\title{
Avaliação da eletrocardiografia de alta resolução em cães clinicamente normais
}

Wagner Luis FERREIRA ${ }^{1}$

Fernanda Gosuen Gonçalves

DIAS $^{2}$

Aparecido Antonio

$\mathrm{CAMACHO}^{1}$

Correspondência para:

WAGNER LUISFERREIRA

Conselho de Ensino, Pesquisa e Extensão

Reitoria- Universidade de Franca

Av. Dr. Armando SalesOliveira, 201 - Parque

Universitário

14403-000-Franca- SP

wluis@francanet.com.br

Recebido para publicação: 30/10/2005 Aprovado para publicação: 15/03/2005

1- Departamento de Clinica e Cirurgia Veterinária da Faculdade de Ciências Agrárias e Veterinária, FCAV - Unesp, Campus de Jaboticabal, Jaboticabal - SP

2- Faculdade de Medicina Veterinária da Universidade de Franca -

UNIFRAN - Franca - SP

\section{Resumo}

No presente estudo, descreve-se a técnica e os resultados obtidos da eletrocardiografia de alta resolução (ECGAR) em cães clinicamente normais. Durante a pesquisa, foram utilizados 32 cães, adultos, sem raça definida, machos e fêmeas, divididos em quatro grupos de acordo com o peso corporal médio, sendo submetidos ao estudo da ECGAR no domínio do tempo. Quanto aos parâmetros eletrocardiográficos analisados, foi constatado que a duração do complexo QRS foi menor no grupo de cães com peso corporal inferior a 10 quilogramas. Por sua vez, a duração dos sinais de baixa amplitude dos últimos $40 \mathrm{~ms}$ do complexo QRS (LAS 40) não alterou significativamente entre os grupos. O mesmo ocorreu em relação à raiz quadrada média da voltagem ao quadrado dos últimos $40 \mathrm{~ms}$ do complexo QRS (RMS 40), permanecendo sem alteração significativa. Os resultados encontrados uma vez tabulados poderão contribuir na padronização dos valores para a espécie canina e servirão de subsídio para outras pesquisas com cães propensos ao desenvolvimento de arritmias ventriculares sustentadas e morte súbita, por meio da detecção dos chamados potenciais tardios.

\section{Introdução}

Arritmias cardíacas são desordens no ritmo cardíaco, muitas vezes observadas em pacientes com doença cardiovascular. $\mathrm{O}$ desenvolvimento das arritmias ventriculares pode precipitar ou intensificar quadros clínicos de insuficiência cardíaca congestiva. Dentre as arritmias cardíacas, a taquicardia ventricular sustentada e a fibrilação ventricular são causas comuns de morte em cães. A redução da incidência de morte por estas arritmias requer a identificação, de forma mais precisa, de pacientes sob alto risco para estes eventos.

Neste sentido, a eletrocardiografia de alta resolução (ECGAR) é um método computadorizado, não invasivo, que serve para identificar pacientes que possuem alto risco de apresentar taquicardia ventricular sustentada por meio da detecção dos chamados potenciais tardios.

Por sua vez, os potenciais tardios são sinais de baixa amplitude e alta freqüência que ocorrem na porção terminal do complexo QRS e/ou início do segmento ST e que estão relacionados com a presença de atividade elétrica fragmentada e retardada nos ventrículos, não sendo evidentes no eletrocardiograma convencional. Tais potenciais são, portanto, marcadores não invasivos de substrato anatômico arritmogênico, que possibilitam a gênese de taquicardia ventricular sustentada por mecanismo de reentrada.

Uma cardiopatia pode produzir áreas de tecidos não excitáveis que criam caminhos para movimento em círculo do impulso elétrico cardíaco. $O$ impulso passa a ser conduzido lentamente ao longo de um caminho que é funcionalmente ou anatomicamente isolado, criando um bloqueio 
1 Modelo CSK 540D - Cardio Sistemas Com. Ind. Ltda. 2 Eletrodos espuma para ECG com gel EMBRAMAC unidirecional. Esta condução isolada pode prover uma via onde o impulso elétrico volta a excitar tecidos que foram previamente estimulados produzindo batimentos prematuros por reentrada.

O valor da ECGAR em identificar pacientes humanos com taquicardia ventricular sustentada tem sido confirmada mormente nos casos de cardiomiopatia dilatada não isquêmica ${ }^{1}$.

Os valores de normalidade do ECGAR ainda não foram totalmente estabelecidos em medicina veterinária e o uso para cães permanece limitado. $\mathrm{Na}$ literatura, verifica-se apenas o trabalho de Calvert ${ }^{2}$, que descreve os valores de normalidade em cães da raça doberman pinscher

Visando contribuir na padronização da técnica do ECGAR em cães, a pesquisa objetiva definir os valores da técnica em cães com variações de peso corporal.

\section{Materiais e Métodos}

\section{Animais}

Foram utilizados 32 animais da espécie canina, adultos, machos ou fêmeas, sem raça definida, considerados clinicamente sadios.

\section{Delineamento experimental}

Para o estudo das variáveis de interesse o total de animais selecionados foi dividido em quatro grupos de oito animais (G1, G2, G3 e G4):

GRUPO 1 (G1)- animais com peso corporal médio inferior a $10 \mathrm{~kg}$.

GRUPO 2 (G2)- animais com peso corporal médio entre 11 e $20 \mathrm{~kg}$.

GRUPO 3 (G3)- animais com peso corporal médio entre 21 e $30 \mathrm{~kg}$.

GRUPO 4 (G4)- animais com peso corporal médio acima de $31 \mathrm{~kg}$

Cada grupo foi constituído por igual número de machos e fêmeas.

Para o registro da ECGAR utilizouse um Kit de análise de Holter digital com software de ECGAR ${ }^{1}$. Os animais foram mantidos em decúbito lateral esquerdo

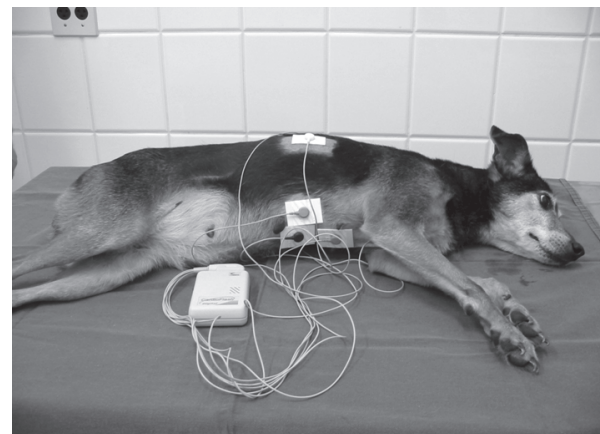

Figura 1 - Animal em decúbito lateral esquerdo submetido a um registro da eletrocardiografia de alta resolução (ECGAR)

(Figura 1), sendo registradas três derivações ortogonais, $\mathrm{X}, \mathrm{Y}$ e Z:

$\mathrm{X}$ : entre o $5^{\circ}$ e $6^{\circ}$ espaço intercostal direito (eletrodo negativo) e esquerdo (eletrodo positivo) na junção entre o terço superior e médio do tórax;

Y: posicionados na região do manúbrio (eletrodo negativo) e cartilagem xifóide (eletrodo positivo);

Z: sobre o processo espinhoso de T7 (eletrodo negativo) e no aspecto oposto ventral (eletrodo positivo).

Os animais foram mantidos em uma sala silenciosa, com pouca luminosidade, sendo realizada uma tricotomia prévia para colocação dos eletrodos. Utilizaram-se eletrodos adesivos com gel' ${ }^{2}$. Por sobre o eletrodo foi colocado uma tira de esparadrapo para conferir maior adesão à pele.

No que se refere à obtenção e processamento dos sinais eletrocardiográficos, observou-se a seguinte seqüência:

1 - registro a partir de três derivações ortogonais $(\mathrm{X}, \mathrm{Y}, \mathrm{Z})$, as quais permitiram que fossem medidos os potencias cardíacos nos planos sagital, transversal e horizontal;

2 - registro, em média, de 1500 ciclos cardíacos, sendo rejeitados os complexos aberrantes ou com muito artefato;

3 - amplificação de cada derivação, 100 vezes para voltagem e quatro vezes para a escala de tempo;

4 - processamento das derivações $X$, Y e Z em filtros bidirecionais; 
Tabela 1 - Valores médiose desvios-padrão das variáveis obtidas por meio do registro daEletrocardiografia de Alta Resolução em 32 cães normais, divididos em quatro grupos de acordo com o peso corporal, Jaboticabal, Unesp, 2003

\begin{tabular}{|c|c|c|c|c|}
\hline \multirow{2}{*}{$\begin{array}{l}\text { Peso } \\
\text { Corporal }\end{array}$} & \multicolumn{4}{|c|}{ Variáveis } \\
\hline & $\begin{array}{l}\text { QRS } \\
\text { (ms) }\end{array}$ & $\begin{array}{l}\text { LAS40 } \\
(\mathrm{ms})\end{array}$ & $\begin{array}{c}\text { RMS40 } \\
(\mu \mathrm{V})\end{array}$ & $\begin{array}{c}\text { Nível de Ruído } \\
(\mu \mathrm{V})\end{array}$ \\
\hline$<10 \mathrm{Kg}$ & $\begin{array}{c}63,75 \pm 6,63 \\
B\end{array}$ & $\begin{array}{c}16,25 \pm 4,26 \\
A\end{array}$ & $359,75 \pm \frac{209,82}{A}$ & $0,42 \pm 0,08$ \\
\hline 11 a $20 \mathrm{Kg}$ & $\begin{array}{c}68,75 \pm 3,53 \\
\mathrm{AB}\end{array}$ & $\underset{\mathrm{A}}{17,12 \pm 4,18}$ & $\begin{array}{c}316,00 \pm 78,12 \\
\mathrm{~A}\end{array}$ & $0,42 \pm 0,10$ \\
\hline 21 a $30 \mathrm{Kg}$ & $\begin{array}{c}73,87 \pm 3,35 \\
\mathrm{~A}\end{array}$ & $\underset{\mathrm{A}}{18,87 \pm 4,58}$ & $\underset{A}{215,62 \pm 62,36}$ & $0,42 \pm 0,13$ \\
\hline$>40 \mathrm{Kg}$ & $\begin{array}{c}74,75 \pm 5,00 \\
\mathrm{~A}\end{array}$ & $\frac{18,50 \pm 3,54}{A}$ & $219,12 \pm \frac{A}{A} 106,13$ & $0,43 \pm 0,15$ \\
\hline
\end{tabular}

Médias seguidas de pelo menos uma mesma letra não são significativamente diferentes (Teste de Tukey, $5 \%$ - $p<0,05$ )

LAS40: duração dos sinais de baixa amplitude ( $<40 \mathrm{mV}$ )

RMS40: raiz quadrada média da voltagem dos últimos 40 ms do complexo QRS

FC: freqüência cardíaca (bpm: batimentos por minuto)

5 - combinação das derivações filtradas em um vetor magnitude (raiz quadrada da somatória $x^{2}+y^{2}+z^{2}$. Tal combinação é uma medida da duração da atividade de alta freqüência de todas as três derivações.

A partir deste processamento, permitiuse a aquisição dos parâmetros avaliados: duração em milisegundos (ms) do complexo QRS filtrado, duração (ms) dos sinais de baixa amplitude (menor que 40 microvolts) dos últimos $40 \mathrm{~ms}$ do complexo QRS (LAS40) e raiz quadrada média da voltagem ao quadrado dos últimos $40 \mathrm{~ms}$ do complexo QRS (RMS40) (Figura 2).

\section{Análise estatística}

Os dados obtidos foram analisados por meio de uma análise de variância e aplicação do teste de Tukey para comparação de médias entre grupos ${ }^{3}$.

\section{Resultados}

No presente estudo, toda eletrocardiografia de alta resolução foi realizada no domínio do tempo, sendo rejeitados os exames com nível de ruído superior a 0,7 microvolts $(\mathrm{mV})$. Foram utilizados filtros bidirecionais $(40 \mathrm{a} 250 \mathrm{~Hz}$ ) com a finalidade de separar os sinais cardíacos de baixa freqüência e a atividade elétrica não cardíaca (atividade muscular e respiratória).

Quanto aos parâmetros eletrocardiográficos analisados, foi constatado que a duração do complexo QRS filtrado foi menor no grupo de cães com peso inferior a $10 \mathrm{~kg}$. (Tabela 1), mostrando uma diferença estatisticamente significativa em relação aos grupos com peso corporal superior a $21 \mathrm{~kg}$. Os valores não diferiram significativamente entre os grupos com peso corporal acima de $11 \mathrm{~kg}$.

Por outro lado, a duração dos sinais de baixa amplitude dos últimos $40 \mathrm{~ms}$ do complexo QRS (LAS40) não alterou significativamente entre

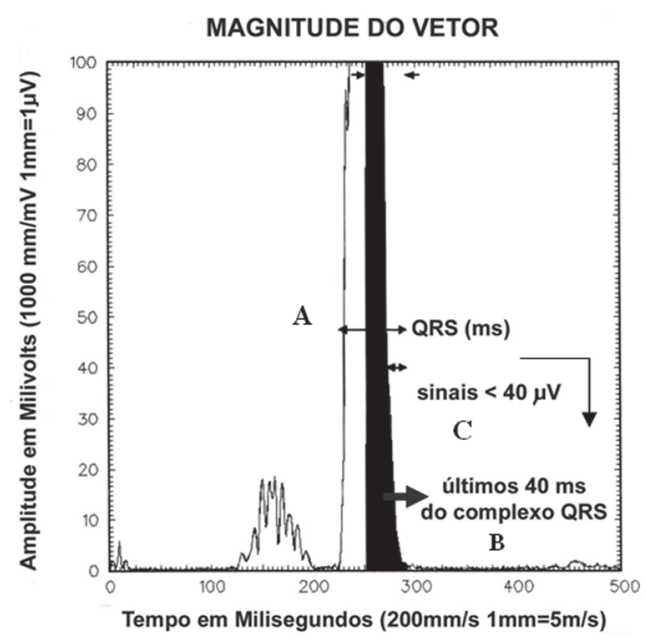

Figura 2 - Características de um traçado de ECGAR analisado no domínio do tempo, em um cão normal. A: duração do QRS filtrado (66 ms); B: duração dos sinais de baixa amplitude (menor que $40 \mathrm{mV}$ ) dos últimos $40 \mathrm{~ms}$ do complexo QRS (19ms); C: raiz quadrada média da voltagem ao quadrado dos últimos 40 ms do complexo QRS (382 $\mu \mathrm{N})$ 
os grupos (Tabela 1). O mesmo ocorreu em relação à raiz quadrada média da voltagem ao quadrado dos últimos $40 \mathrm{~ms}$ do complexo QRS (RMS40), permanecendo sem alteração significativa. O nível de ruído também não alterou significativamente entre os grupos.

$\mathrm{Na}$ avaliação dentro de cada grupo, não ocorreu diferença significativa nos parâmetros analisados.

\section{Discussão}

No presente estudo, a rejeição dos exames com nível de ruído superior a $0,7 \mathrm{mV}$ obedeceu à recomendação da Americam Heart Association ${ }^{4}$. Excessiva atividade muscular pode produzir registros eletrocardiográficos inaceitáveis. Uma adequada redução no ruído é crucial para a análise do ECGAR. Assim, visando reduzir o nível de ruído, os animais permaneceram em uma sala com pouca luminosidade, com um período prévio de permanência junto à mesma. Outros pesquisadores ${ }^{5}$ avaliaram os efeitos da sedação com acepromazina e buprenorfina nos índices da eletrocardiografia de alta resolução em cães normais, evidenciando que a sedação auxiliou na obtenção dos registros da ECGAR sem alterar os índices eletrocardiográficos.

O estudo da eletrocardiografia de alta resolução ainda é de uso limitado em cães. O principal limite para seu uso é a falta de valores normais estabelecidos para a espécie canina.

Baseando-se nos parâmetros da normalidade descritos na literatura ${ }^{2}$, nenhum animal, no presente estudo, evidenciou a ocorrência de potenciais tardios. Tais potenciais são os sinalizadores patofisiológicos de um circuito de reentrada ${ }^{6}$. A ocorrência do potencial tardio pode prolongar a duração do QRS filtrado e do LAS40. Além disso, pode ocorrer uma diminuição na amplitude do RMS40. Admite-se a presença de potencial tardio quando dois ou mais dos parâmetros analisados (QRS, LAS40 e RMS40) mostrarem-se anormais?

Vale ressaltar, que os valores da normalidade dos parâmetros da eletrocardiografia de alta resolução descritos na literatura são específicos a cães da raça doberman pinscher.

Os resultados da variação pertinentes a duração do complexo QRS filtrado corroboram com os relatos de Tilley ${ }^{8}$, que descreveu os valores da normalidade da eletrocardiografia convencional em cães. Segundo este autor, a duração do QRS em raças caninas de pequeno porte é inferior em relação a raças de grande porte. Neste sentido, o padrão de normalidade da duração do QRS filtrado na ECGAR descrito por Calvert ${ }^{2}$ não deve ser aplicado a raças caninas de pequeno porte com peso corporal inferior a $10 \mathrm{Kg}$.

Por outro lado, a falta de variação significativa nos valores da LAS40 e RMS40 entre os grupos avaliados nessa pesquisa sugere que os padrões de normalidade descritos na literatura relativos a esses parâmetros aplicamse a cães com diferentes pesos corporais.

Em síntese, os resultados obtidos no presente estudo contribuem na padronização da eletrocardiografia de alta resolução em cães normais, propiciando subsídios na interpretação e detecção de pacientes propensos ao desenvolvimento de arritmias ventriculares e morte súbita.

\section{Agradecimentos}

Agradecemos à Fundação de Amparo a Pesquisa do Estado de São Paulo (FAPESP) pela concessão do auxílio à pesquisa.

\section{High resolution electrocardiography avaliation in clinically healthy dogs}

\section{Abstract}

The aim of this study was to describe the technique and results of high resolution electrocardiography (ECGAR) in clinically healthy dogs. During this research, 32 adult crossed-breed, dogs from both
Key-words:

High resolution electrocardiography. Late potentials. Dogs. 
sexes were used, which were divided in four groups accordingly to their mean body weight. The animals were submitted to high resolution electrocardiography in time domain. It was verified that the duration of QRS complex was smaller in the group of dogs with lower body weight when compared to the group of animals weighing over 21 kilograms. Moreover, the duration of the low amplitude signals of the last $40 \mathrm{~ms}$ of the QRS complex (LAS 40) did not show significant differences among groups. Also no significant difference was seen among groups in relation to the mean squared root of the squared voltage of the last $40 \mathrm{~ms}$ of the QRS complex (RMS 40). These results, once tabled, could contribute for standardization of the reference values for canine ECGAR. Furthermore, these values could serve as foundation for other researches with dogs in risk of development of sustained ventricular arrhythmias and sudden death, through the detection of the so called late potentials.

\section{Referências}

1 LINDSAY, B. D. Detection of patients with nonischemic cardiomyopathy prone to sustained ventricular arrhythmias by frequency analysis of signal averaged electrocardiograms. Circulation v. 6, p. 336345, 1987.

2 CALVERT, C. A. Hight resolution electrocardiography. In: GOODWIN, J. The veterinary clinics of north america small animal practice - advances in cardiovascular diagnostics and therapy. Philadelphia: W.B. Saunders, 1998. n. 8, p. 1429-1447.

3 ZAR, J. Biostatistical analyisis. 4. ed. New Jersey: Prentice Hall, 1999, p. 161-163.

4 DUNBAR, D. N.; DENES, P. Operational aspects of signal-averaged electrocardiography. Progress in Cardiovasscular Diseases, v. 35, n. 5, p. 329-348, 1993.

5 KUSHNER, L. I.; CALVERT, C. A.; BOYLE, C. R. Effects os acepromazine and buprenorphine on measured of the signal-averaged electrocardiogram in healthy dogs. American Journal of Veterinary Research, V. 57, n. 10, p. 1511-1514, 1996.

6 BERBARI, E. J. et al. Recording from the body surface of arrhythmogenic ventricular activity during the S-T segment. American Journal of Cardiology, v. 41, n. 4, p. 697-702, 1978.

7 MOFFA, P. J. Eletrocardiograma de alta resolução. In: FRIEDMANN, A. A.; GRINDLER, J. (Ed.) Eletrocardiografia básica. São Paulo: [s. n.], 2000. p. 124.

8 TILLEY, L. P. Essentials of canine and feline eletrocardiography. 3. ed. Philadelphia: Lea \& Febiger, 1992. $470 \mathrm{p}$. 